\title{
The First Photoexcitation Step of Ruthenium-Based Models for Artificial Photosynthesis Highlighted by Resonance Raman Spectroscopy
}

\section{Supplementary Material}

Carmen Herrmann ${ }^{\dagger}$, Johannes Neugebauer ${ }^{*}$, , Martin Presselt ${ }^{\ddagger}$, Ute Uhlemann $^{\ddagger}$, Michael Schmitt ${ }^{\ddagger}$, Sven Rau ${ }^{\diamond}$, Jürgen Popp ${ }^{\ddagger}$, and Markus Reiher $^{*} \dagger$

${ }^{\dagger}$ Laboratorium für Physikalische Chemie, ETH Zürich, Wolfgang-Pauli-Str. 10, CH-8093 Zürich, Switzerland

${ }^{\ddagger}$ Institut für Physikalische Chemie, Friedrich-Schiller-Universität Jena, Helmholtzweg 4, D-07743 Jena, Germany

${ }^{\diamond}$ Institut für Anorganische Chemie, Friedrich-Schiller-Universität Jena August-Bebel-Str. 2, D-07743 Jena, Germany

* To whom correspondence should be addressed, email: \{markus.reiher, johannes.neugebauer\}@phys.chem.ethz.ch, FAX: +41-44-63-31594, TEL: +41-44-63-34308

Date: $\quad$ March 1st, 2007

Status: submitted as a full paper to J. Phys. Chem. B 

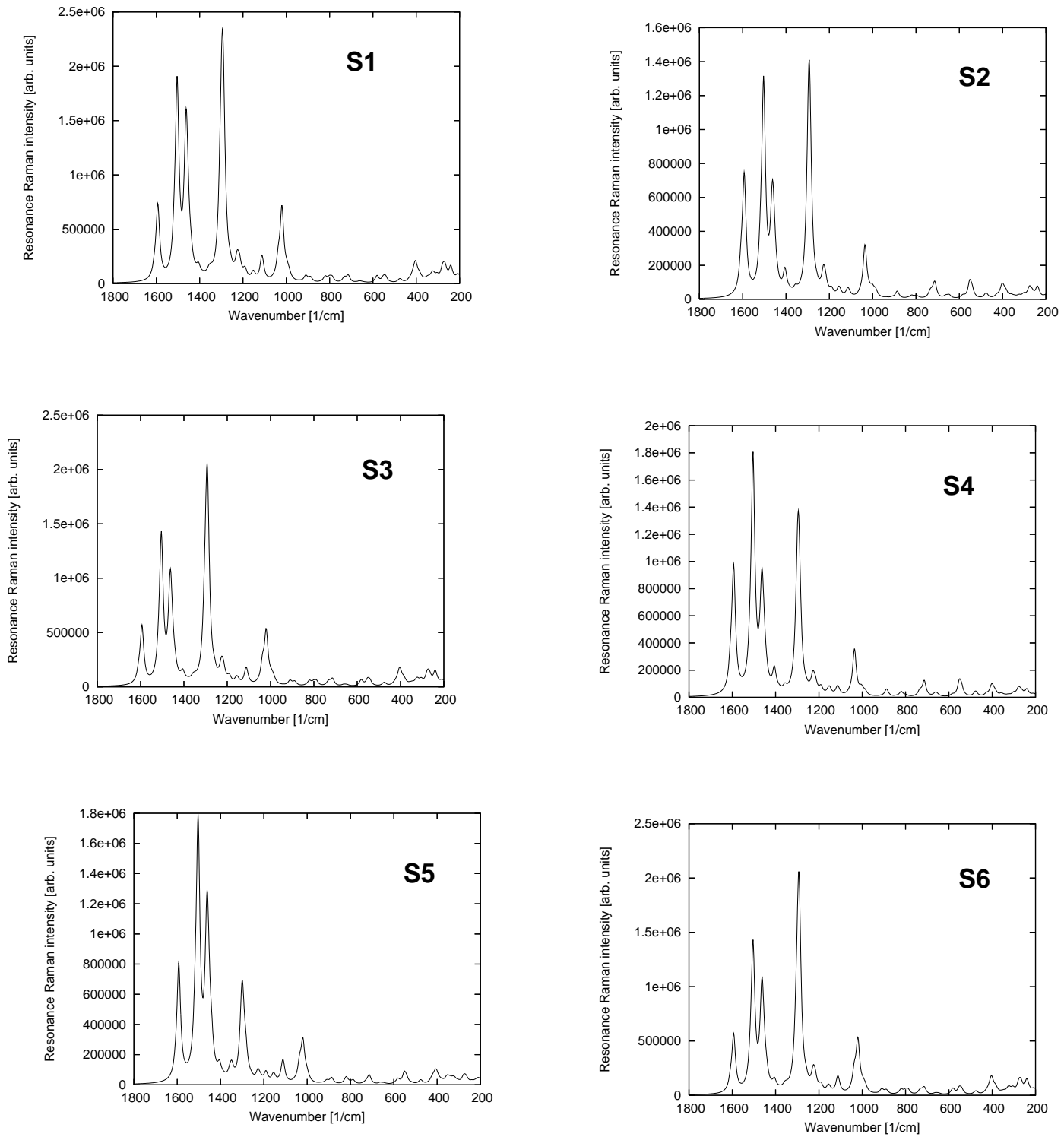

Figure 1: Calculated resonance Raman spectra for the $\left[\mathrm{Ru}_{2} \mathrm{Pd}_{2}\right]$ complex $\mathbf{2}$ assuming excitation to one of the 6 lowest-lying singlet states each. Vibrational normal modes, frequencies, and resonance Raman intensities: BP86(RI)/TZVP. Spectra plotted with Gaussian line-broadening, using a halfwidth of $20 \mathrm{~cm}^{-1}$. 


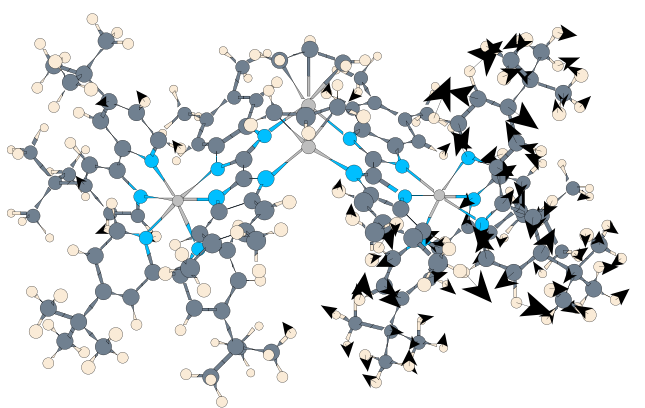

$1502 \mathrm{~cm}^{-1}(\mathrm{~S} 1,2)$

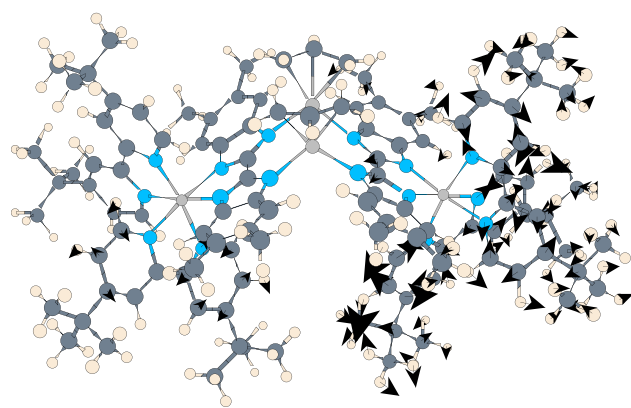

$1504 \mathrm{~cm}^{-1}$ (S 1,2)

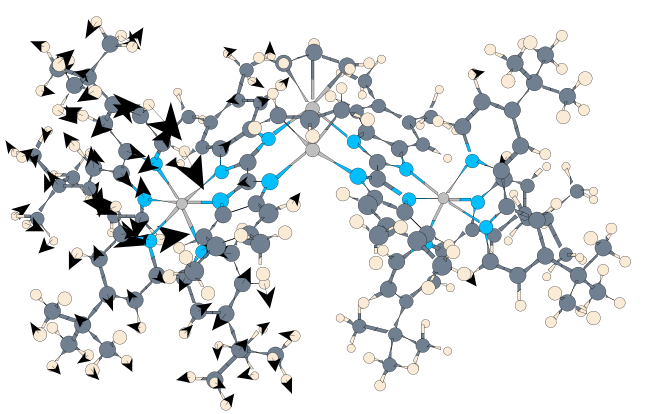

$1503 \mathrm{~cm}^{-1}(\mathrm{~S} 3,4)$

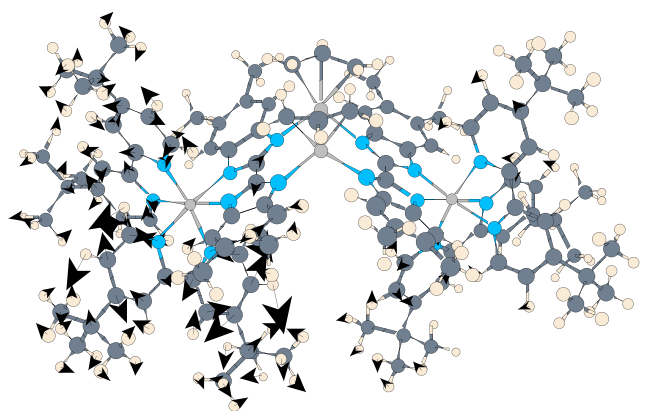

$1505 \mathrm{~cm}^{-1}(\mathrm{~S} \mathrm{3,4)}$

Figure 2: Normal modes responsible for the some of the most intense peaks in the $\left[\mathrm{Ru}_{2} \mathrm{Pd}_{2}\right](\mathbf{2})$ resonance Raman spectra calculated assuming different low-lying singlet excitations (indicated by S...). 


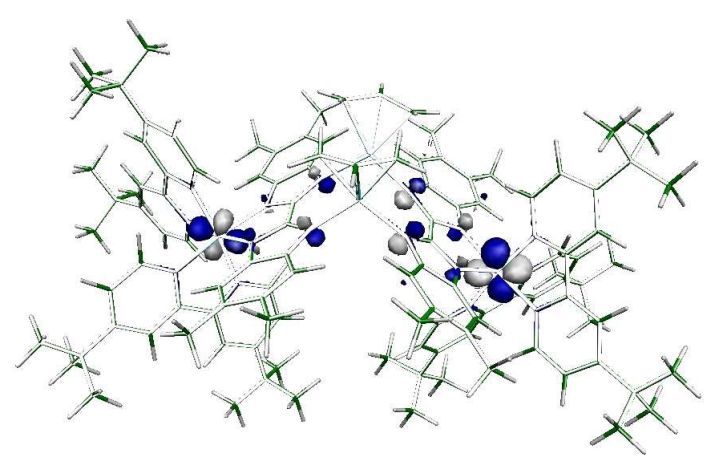

HOMO-3 (- 0.2811 a.u.)

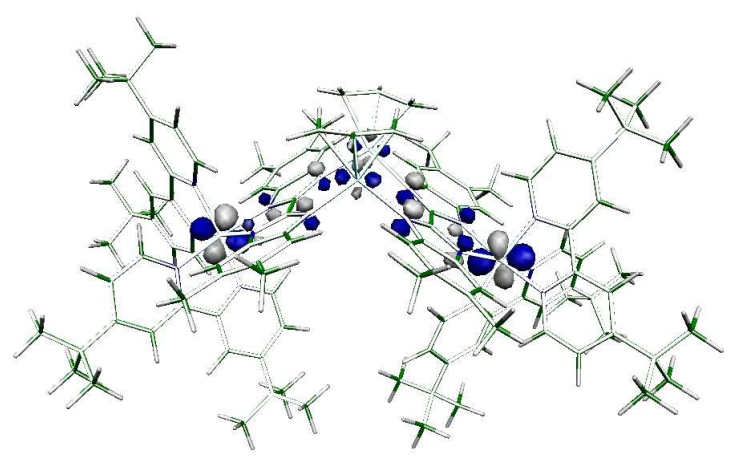

HOMO-2 (-0.2778 a.u.)

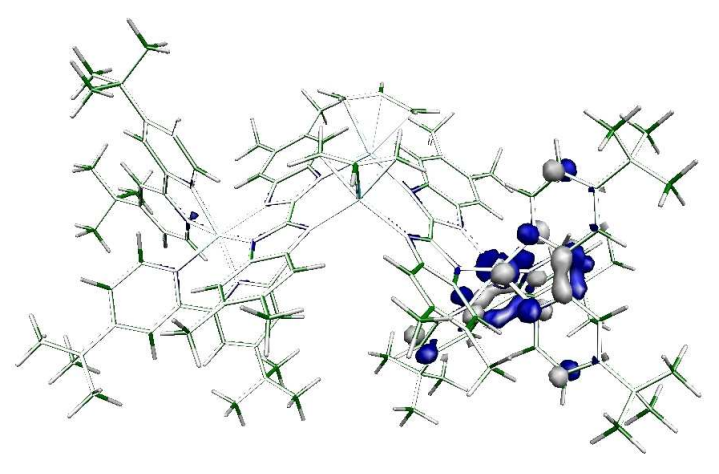

LUMO+2 (-0.2243 a.u.)

Figure 3: Further molecular orbitals important for the excitations to the lowest excited singlet states in the $\left[\mathrm{Ru}_{2} \mathrm{Pd}_{2}\right]$ complex 2 . The corresponding orbital energies are given in parentheses. Ground-and excited-state calculations with BP86(RI)/TZVP. 


\begin{tabular}{|c|c|c|c|c|c|}
\hline transition & $\begin{array}{l}\text { transition dipole } \\
\text { (length rep.) / Db }\end{array}$ & $\begin{array}{c}\text { transition } \\
\text { energy / eV }\end{array}$ & $\begin{array}{l}\text { transition } \\
\text { energy / nm }\end{array}$ & mainly contributing MOs & contr. / \% \\
\hline 1 & 0.023 & 1.203 & 1030 & $\mathrm{HOMO} \rightarrow \mathrm{LUMO}$ & 96.3 \\
\hline \multirow[t]{2}{*}{2} & 0.761 & 1.233 & 1005 & HOMO-1 $\rightarrow$ LUMO & 83.9 \\
\hline & & & & $\mathrm{HOMO}-1 \rightarrow \mathrm{LUMO}+1$ & 11.0 \\
\hline \multirow[t]{2}{*}{3} & 0.367 & 1.237 & 1002 & $\mathrm{HOMO}-1 \rightarrow \mathrm{LUMO}+1$ & 82.5 \\
\hline & & & & HOMO-1 $\rightarrow$ LUMO & 11.4 \\
\hline 4 & 0.672 & 1.255 & 988 & $\mathrm{HOMO} \rightarrow \mathrm{LUMO}+1$ & 92.4 \\
\hline \multirow[t]{2}{*}{5} & 0.648 & 1.324 & 936 & HOMO-2 $\rightarrow$ LUMO & 89.9 \\
\hline & & & & HOMO-3 $\rightarrow$ LUMO & 7.3 \\
\hline 6 & 0.623 & 1.346 & 921 & $\mathrm{HOMO}-2 \rightarrow \mathrm{LUMO}+1$ & 90.0 \\
\hline \multirow[t]{2}{*}{7} & 0.089 & 1.361 & 911 & $\mathrm{HOMO} \rightarrow \mathrm{LUMO}+3$ & 77.0 \\
\hline & & & & $\mathrm{HOMO} \rightarrow \mathrm{LUMO}+2$ & 18.8 \\
\hline \multirow[t]{2}{*}{8} & 0.076 & 1.362 & 910 & $\mathrm{HOMO}-1 \rightarrow \mathrm{LUMO}+2$ & 83.4 \\
\hline & & & & $\mathrm{HOMO}-1 \rightarrow \mathrm{LUMO}+3$ & 11.2 \\
\hline \multirow[t]{3}{*}{9} & 0.092 & 1.393 & 890 & $\mathrm{HOMO} \rightarrow \mathrm{LUMO}+2$ & 68.3 \\
\hline & & & & $\mathrm{HOMO} \rightarrow \mathrm{LUMO}+3$ & 16.3 \\
\hline & & & & $\mathrm{HOMO}-3 \rightarrow \mathrm{LUMO}+1$ & 8.3 \\
\hline \multirow[t]{3}{*}{10} & 0.039 & 1.397 & 887 & $\mathrm{HOMO}-1 \rightarrow \mathrm{LUMO}+3$ & 52.8 \\
\hline & & & & HOMO-3 $\rightarrow$ LUMO & 36.3 \\
\hline & & & & $\mathrm{HOMO}-1 \rightarrow \mathrm{LUMO}+2$ & 7.5 \\
\hline
\end{tabular}

Table 1: Lowest-lying singlet transitions for the $\left[\mathrm{Ru}_{2} \mathrm{Pd}_{2}\right]$ complex 2 (BP86(RI)/TZVP). Note that the long-range charge transfer transitions (like HOMO $\rightarrow$ LUMO) feature small transition dipole moments (but note in the case of the 3rd singlet transition that the transition dipole moment is significantly different from zero because of the HOMO-1 $\rightarrow$ LUMO contribution (which is not of long-range charge transfer type) resulting from a near degeneracy with transition 2). Note that the transition energies for the long-range charge transfer excitations 1 and 3 calculated within a TDDFT framework are very similar to the orbital-energy differences of those orbital pairs that are the mainly contributing one-particle transitions. This equivalence is a general feature of long-range charge transfer excitations within local density approximation and generalized gradient approximation DFT calculations. The orbital-energy differences for the mainly contributing orbital pairs of the first four transitions are $1.203 \mathrm{eV}, 1.211 \mathrm{eV}, 1.238 \mathrm{eV}$, and $1.230 \mathrm{eV}$. 